JHR

35,4

\section{8}

Received 24 June 2020

Revised 26 August 2020

9 September 2020

Accepted 15 September 2020

\title{
COVID-19 - what do we know and how are we dealing with it? A quick online cross-sectional study in India
}

\author{
Ashwani Verma \\ Campbell South-Asia, New Delhi, India \\ Dhanshree R Gunjawate
}

Department of Audiology and Speech Language Pathology, Kasturba Medical College, Mangalore, Manipal Academy of Higher Education, Manipal, India

Sanny Bhushan Kumar

Vedanta Medical Research Foundation (BALCO Medical Center), Raipur, India

CNV Sai Bharath

Jhpiego, Lucknow, India, and

Rohit Ravi

Department of Audiology and Speech Language Pathology, Kasturba Medical College, Mangalore, Manipal Academy of Higher Education, Manipal, India

\begin{abstract}
Purpose - Coronavirus disease 2019 is a global public health emergency affecting almost the entire world. The exponential spread of the infection has become a matter of concern. The study aims to determine how well people in India know about COVID-19 outbreak and actions taken to cope up during lockdown.

Design/methodology/approach - A total of 5,000 randomly selected participants were sent the survey link, of which 1,526 responded (response rate of 30.5\%). The survey was conducted from March 28 till March 31,2020 . The 20 -item questionnaire aimed to determine how well people know about COVID-19 outbreak as well as actions taken to cope up during the lockdown. Descriptive statistics were used to summarize the findings.

Findings - Participants exhibited good knowledge about the primary and secondary symptoms of COVID-19 and infection spread. Over 95\% participants knew preventive measures such as use of masks, sanitizers and hand wash. And, $80 \%$ of the participants felt that steps taken by the government are effective, while $93 \%$ opined that lockdown would be effective. There was apprehension regarding the infection spread, its containment and age group affected. Over 60\% referred the World Health Organization website as their source of information. During the lockdown, participants engaged in homebased exercise $(39 \%)$ and yoga $(28 \%)$ for fitness while used social media $(64 \%)$ and television $(63 \%)$ for entertainment.

Originality/value - Although participants exhibited good knowledge related to symptoms and preventive measure related to COVID-19, the beliefs related to infection spread and containment need to be addressed.
\end{abstract}

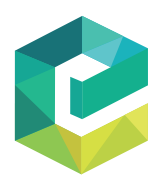

Journal of Health Research Vol. 35 No. 4,2021 pp. 298-306

Emerald Publishing Limited e-ISSN: $2586-940 \mathrm{X}$ p-ISSN: 0857-4421 DOI 10.1108/JHR-06-2020-0231
(C) Ashwani Verma, Dhanshree R Gunjawate, Sanny Bhushan Kumar, CNV Sai Bharath and Rohit Ravi. Published in Journal of Health Research. Published by Emerald Publishing Limited. This article is published under the Creative Commons Attribution (CC BY 4.0) licence. Anyone may reproduce, distribute, translate and create derivative works of this article (for both commercial and non-commercial purposes), subject to full attribution to the original publication and authors. The full terms of this licence may be seen at http://creativecommons.org/licences/by/4.0/legalcode

The authors thank all the participants for participating in this study. The authors thank Garima Verma and Nikita Srivastava for their support. 
These should be targeted through health promotional campaigns focusing on mental health and home-based physical activities.

Keywords COVID-19, Coronavirus, Lockdown, India

Paper type Research paper

COVID-19

A quick online survey

\section{Introduction}

A local outbreak of pneumonia that was initially thought to be of unknown origin was detected in Wuhan (Hubei, China) in December 2019. This outbreak was determined to have begun due to a novel virus, which was subsequently named as severe acute respiratory syndrome coronavirus 2 (SARS-CoV-2) or coronavirus disease 2019 (COVID-19) [1]. Subsequently, it spread to other provinces in Mainland China as well as other countries. The outbreak of COVID-19 was confirmed as a Public Health Emergency of International Concern (PHEIC) [1] at the meeting of the Emergency Committee of the World Health Organization (WHO) on January 30, 2020. Further, the WHO declared it as a "pandemic" on March 11, 2020 [2]. COVID-19 with an incubation period of 0-14 days spread rapidly across the world, with over 1.17 million cases as on April 6, 2020 [3].

In India, on January 31, 2020, the first case of positive COVID-19 was reported in an individual with a recent travel history from China. The situation report dated February 28, 2020 , indicated no new cases. However, the number escalated from nine cases on March 9, to 3,577 cases as on April 6,2020 [4]. To contain this spread of the virus, the Government of India (GoI) announced a countrywide lockdown starting at midnight on March 24, 2020 [5].

To encourage the population to remain calm in these nerve-wracking situations, the GoI has taken further measures such as urging people to stay at home barring essential needs, spreading importance toward hand hygiene and social distancing. The use of several platforms such as social media, news, television, radio and the internet has been used to reach maximum population. The government has also made available answers to frequently asked questions on the Ministry of Health and Family Welfare (MoHFW) GoI website [6].

The lockdown and the restrictions imposed have been well received and appreciated globally as being comprehensive, robust and timely [5]. However, it might have led to increased apprehension and anxiety among the masses. It is, thus, crucial to understand how aware are the people about the nature of COVID-19 as well as the measures that they are implementing in containing the spread. Besides, it would be interesting to know the steps being taken toward physical fitness as well as to keep oneself entertained. The present study aimed toward determining how well people in India know about COVID-19 outbreak and actions toward coping with the lockdown.

\section{Material and methods}

Survey participants

An online cross-sectional survey was used to collect the information about how well people know about COVID-19 outbreak and actions toward coping with the lockdown using snowball sampling. COVID-19-related online survey was excluded from ethical approval during national lockdown. The participation was voluntary, and informed consent from each participant was taken prior to the survey. The questionnaire was sent to approximately 5,000 participants via sharing a link of Google Forms, the identity of the contributors were kept anonymous. The minimum eligibility to participate in the survey was that they should have attained at least 18 years of age and should read and understand the English language. The exclusion criteria excluded participants who do not consent to participate and were digitally illiterate.

Sample size calculation. For estimation of proportion:

$$
n=\frac{Z_{1-\alpha / 2}^{2} P(1-P)}{d^{2}}
$$


JHR

35,4

300

$Z_{1-\alpha / 2}=1.96$ (value at 95\% CI), $P=50 \%$ (knowledge and awareness data regarding COVID-19 among Indian population is unknown, therefore, prevalence is assumed to be $50.0 \%$ for this study), $d=0.026$ (acceptable margin of error in estimating true population proportion), $n=1,420$ (sample size).

Survey tool. The questionnaire used in the present study was developed by referring information about COVID-19 from the website of WHO [7], Centers for Disease Control and Prevention (CDC) [8] and discussion with the experts based on their experience/opinion. The developed questionnaire was provided to three public health expert, with more than ten years of work and research experience. The experts were asked to rate each question on a rating scale of relevant, somewhat relevant, somewhat irrelevant and irrelevant. Only items rated as relevant and somewhat relevant were included. All the questions that were proposed in the initial version were included. An overall score of 0.8 was obtained on the content validity index for scale (S-CVI) for the questionnaire, indicative of a very good content validity. [9] The questionnaire was then piloted among five public health professionals with more than five years of experience for content validity from different states of India. The questionnaire consisted of 20 items. The response options included multiple-choice, checkboxes and short answers. The components of the questionnaire used were as follows: symptoms related to COVID-19 (two items), transmission of infection and incubation period (three items), preventive measures (six items), strategies against COVID-19 (three items), beliefs related to COVID-19 (two items), groceries at home during lockdown (one item), engagement in physical activities during lockdown (one item), entertainment during lockdown (one item) and sources of information (one item).

Data collection. The data collection link was kept active for three days, starting from March 28 till March 31. 2020. Snowball sampling was used for data collection. The questionnaire was administered after the complete lockdown was announced by the GoI. The details of the study were provided to the participants as a note prior to their participation in the survey. The questionnaire was administered in the English language.

Data analysis. The data analysis was done using descriptive statistics of mean and standard deviation for continues variables, while for discrete variables, frequency and percentage was used. The responses obtained in the study were analyzed using SPSS-15.

Ethical consideration. The study was conducted in accordance with the Helsinki declaration [10]. The institutional ethics committee permitted online surveys on COVID-19 during the lockdown. Prior consent was taken from the participant before participation in the survey, and their participation was voluntary.

\section{Results}

A total of 5,000 randomly selected participants were sent the survey link, of which 1,526 responded (response rate of $30.5 \%$ ). Ten respondents did not wish to participate in the study, while 1,516 complete responses were recorded.

The mean age of the participants was 28.70 years $( \pm 8.90)$, range $18-84$ years. More than half, $51.3 \%$ were female participants, and $47.9 \%$ were male participants and $0.9 \%$ participants preferred not to reveal their gender. Around $40 \%$ of participants belonged to the age group of 25-31 years. Educational profile of the participants depicted that maximum participants held a minimum of graduate degree and above $(84.3 \%)$. In terms of the occupation, nearly one-third $(33.5 \%)$ were healthcare workers, followed by students $(28.2 \%)$. Table 1 depicts the characteristics of study participants.

\section{Symptoms related to COVID-19}

Almost all participants (95.2\%) reported that the common symptoms of COVID-19 are fever, tiredness and dry cough. Very few $(2.5 \%)$ refute these symptoms as COVID-19 infection, and rest $2.3 \%$ were not sure about these symptoms. When asked for other symptoms (aches, pain, 


\begin{tabular}{|c|c|c|c|c|c|}
\hline & Female & Male & Prefer not to say & Total & \\
\hline \multicolumn{5}{|l|}{ Age } & \multirow{4}{*}{$\begin{array}{r}\text { A quick online } \\
\text { survey }\end{array}$} \\
\hline Mean $\pm \mathrm{SD}$ (years) & $27.80 \pm 8.69$ & $29.71 \pm 8.99$ & $29.23 \pm 13.17$ & $28.70 \pm 8.90$ & \\
\hline Range (years) & $\begin{array}{l}18-74 \\
n(\%)\end{array}$ & $\begin{array}{l}18-84 \\
n(\%)\end{array}$ & $\begin{array}{l}18-69 \\
n(\%)\end{array}$ & $\begin{array}{l}18-84 \\
n(\%)\end{array}$ & \\
\hline Participants & 777 (51.3) & $726(47.9)$ & $13(0.9)$ & $1516(100)$ & \\
\hline \multicolumn{5}{|l|}{ Age group (years) } & 301 \\
\hline $18-24$ & $316(40.7)$ & $188(25.9)$ & $5(38.5)$ & 509 (33.6) & \\
\hline $25-31$ & $289(37.2)$ & $322(44.4)$ & 5 (38.5) & $616(40.6)$ & \\
\hline $32-38$ & 98 (12.6) & $142(19.6)$ & $2(15.4)$ & $242(16)$ & \\
\hline $39-45$ & $28(3.6)$ & $33(4.5)$ & $0(0.0)$ & $61(4)$ & \\
\hline $46-52$ & 26 (3.3) & $14(1.9)$ & $0(0.0)$ & $40(2.7)$ & \\
\hline $53-59$ & $15(1.9)$ & $16(2.2)$ & $0(0.0)$ & $31(2)$ & \\
\hline$>60$ & $5(0.6)$ & $11(1.5)$ & $1(7.7)$ & $17(1.1)$ & \\
\hline \multicolumn{6}{|l|}{ Education levels } \\
\hline Up to class 10 th & $15(1.9)$ & $16(2.2)$ & $0(0.0)$ & $31(2)$ & \\
\hline Intermediate & $68(8.8)$ & $38(5.2)$ & $0(0.0)$ & $106(7)$ & \\
\hline Diploma & $39(5)$ & $61(8.4)$ & $2(15.4)$ & $102(6.7)$ & \\
\hline Graduate & 335 (43.1) & 317 (43.7) & $6(46.2)$ & $658(43.4)$ & \\
\hline Postgraduate & $302(38.9)$ & $274(37.7)$ & $4(30.8)$ & $580(38.3)$ & \\
\hline $\mathrm{PhD}$ & $18(2.3)$ & $20(2.8)$ & $1(7.7)$ & 39 (2.6) & \\
\hline \multicolumn{6}{|l|}{ Occupation } \\
\hline Healthcare worker & $265(34.1)$ & $242(33.3)$ & $1(7.7)$ & $508(33.5)$ & \\
\hline $\begin{array}{l}\text { Government officials other than healthcare } \\
\text { worker }\end{array}$ & $44(5.7)$ & 47 (6.5) & $2(15.4)$ & $93(6.1)$ & \\
\hline $\begin{array}{l}\text { Private workers other than healthcare } \\
\text { worker }\end{array}$ & 87 (11.2) & $186(25.7)$ & $2(15.4)$ & 275 (18.1) & \\
\hline Self-employed/own business & $34(4.3)$ & $93(12.8)$ & $3(23.1)$ & $130(8.6)$ & Table 1. \\
\hline Homemaker & $77(9.8)$ & $2(0.3)$ & $1(7.7)$ & $80(5.3)$ & Characteristics of \\
\hline Student & $269(34.6)$ & 155 (21.3) & $4(30.8)$ & $428(28.2)$ & study participants \\
\hline
\end{tabular}

nasal congestion, running nose, sore throat or diarrhea), more than three-fourth $(80.5 \%)$ responded correctly. These details are depicted in Table 2.

\section{Transmission of the infection and incubation period}

Majority participants (95.0\%) agreed correctly that the COVID-19 is spread through small droplets from nose/mouth of an infected person. Maximum $(80.0 \%)$ participants correctly identified that the use of multiple masks, smoking and consuming alcohol are not preventive measures against COVID-19 infection. Almost 90.0\% correctly answered the question on the incubation period of the COVID-19 to be $0-14$ days. The remaining responses were as follows; $7 \%$ answered $15-20$ days, $2.4 \%$ replied $21-25$ days and $1.1 \%$ responded beyond 25 days.

\section{Preventive measures}

Nearly all participants $(95.4 \%)$ reported that hand washing/sanitizer limits the spread of COVID-19. Only $1.3 \%$ of participants did not agree, while $3.3 \%$ of the participants were not sure about the effect of hand washing/sanitizer. Maximum participants $(95.3 \%)$ were aware of the hand washing techniques. Most of the participants $(62.5 \%)$ were scared of contacting COVID-19 infection. These details are depicted in Table 2.

Majority of the participants $(71.5 \%$ ) felt that the coronavirus could be controlled, but few participants $(6.3 \%)$ felt it might not be controlled, while around $20.0 \%$ were not sure about 
JHR

35,4

\begin{tabular}{|c|c|c|c|}
\hline Knowledge & $\begin{array}{l}\text { Correct } \\
\text { response } n(\%)\end{array}$ & $\begin{array}{l}\text { Incorrect } \\
\text { response } n(\%)\end{array}$ & Not sure $n(\%)$ \\
\hline $\begin{array}{l}\text { Most common symptoms of COVID-19 are fever, } \\
\text { tiredness and dry cough }\end{array}$ & $1,443(95.2)$ & $38(2.5)$ & $35(2.3)$ \\
\hline $\begin{array}{l}\text { Other symptoms of COVID-19 may include aches, } \\
\text { pain, nasal congestion, running nose, sore throat or } \\
\text { diarrhea }\end{array}$ & $1,220(80.5)$ & $123(8.1)$ & $173(11.4)$ \\
\hline $\begin{array}{l}\text { Transmission of COVID-19 can happen through } \\
\text { small droplets from nose or mouth of infected } \\
\text { person }\end{array}$ & $1,440(95.0)$ & $41(2.7)$ & $35(2.3)$ \\
\hline $\begin{array}{l}\text { Wearing multiple masks, smoking and consuming } \\
\text { alcohol are effective against COVID-19 }\end{array}$ & $1,214(80.0)$ & $151(10.0)$ & $151(10.0)$ \\
\hline Awareness & Yes $n(\%)$ & No n (\%) & Not sure $n(\%)$ \\
\hline $\begin{array}{l}\text { Hand washing/sanitizer helps in preventing } \\
\text { COVID-19 spread }\end{array}$ & $1,446(95.4)$ & $20(1.3)$ & $50(3.3)$ \\
\hline Awareness about hand washing technique & $1,444(95.3)$ & $45(3.0)$ & $27(1.8)$ \\
\hline Being scared against COVID-19 infection & 948 (62.5) & $412(27.2)$ & $156(10.3)$ \\
\hline COVID-19 can be controlled successfully & $1,084(71.5)$ & $95(6.3)$ & $337(22.2)$ \\
\hline $\begin{array}{l}\text { Role of health-promotion messages is effective to } \\
\text { contain COVID-19 infection }\end{array}$ & $1,062(70.1)$ & $231(15.2)$ & $223(14.7)$ \\
\hline $\begin{array}{l}\text { Steps taken by government are effective to contain } \\
\text { COVID- } 19 \text { infection }\end{array}$ & $1,210(79.8)$ & $176(11.6)$ & $130(8.6)$ \\
\hline $\begin{array}{l}\text { Lockdown is an effective strategy to limit the } \\
\text { spread of coronavirus }\end{array}$ & $1,414(93.3)$ & $32(2.1)$ & $70(4.6)$ \\
\hline $\begin{array}{l}\text { Coronavirus spread may be limited during summer } \\
\text { or high temperature }\end{array}$ & $337(22.2)$ & $674(44.5)$ & $505(33.3)$ \\
\hline
\end{tabular}

this. When asked for the effect of high temperature/summer on COVID-19 spread, almost $45.0 \%$ participants refute any effect of high temperature on COVID-19 infection. These details are depicted in Table 2.

Nearly half of the participants $(46.1 \%)$ reported that ideal distance that has to be maintained from COVID-19 infected patient is $3 \mathrm{ft}$ as compared to $28.8 \%$ participants reported the ideal distance was $6 \mathrm{ft}$. Very few participants $(3.9 \%)$ reported that the ideal distance was $8 \mathrm{ft}$, and the rest $(21.2 \%)$ reported the ideal distance of $1 \mathrm{ft}$.

\section{Strategies against COVID-19}

More than three-fourth of the participants $(79.8 \%)$ supported Gol's approach to containing COVID-19 infection. However, nearly $10.0 \%$ of the participants were not satisfied by their initiatives, while around $8.0 \%$ were not sure about the effect of these initiatives. Majority of the participants $(70.1 \%)$ reported that the health-promotion messages through mass media were effective in providing correct information on COVID-19 infection. Almost all participants $(93.3 \%)$ agreed that lockdown is an effective strategy to limit the transmission of COVID-19 infection as compared to only $2.1 \%$ who reported otherwise, and the rest of the participants $(4.6 \%)$ were unsure about the lockdown. These details are depicted in Table 2.

\section{Sources of information about COVID-19}

More than half of the participants $(60.0 \%)$ preferred the WHO website as their source of information followed by the internet - Google search (49.0\%), media and newspaper $(47.0 \%)$, GoI websites $(42.0 \%)$ and social media like Facebook, Instagram, Twitter, WhatsApp 
(38.0\%). Less refereed sources were the CDC website $(26.0 \%)$, physician $(10.0 \%)$ and friends $(13.0 \%)$.

COVID-19

A quick online

survey

Beliefs related to COVID-19

Based on their beliefs about death due to COVID-19, most of the participants (59.4\%) felt that death was limited to less than $4.0 \%$ among infected individuals. Around $21 \%$ participants reported death was in between 5.0 and $8.0 \%$ among infected individuals, $10.9 \%$ participants reported deaths in between 9.0 and $12.0 \%$ among infected individuals and nearly $8 \%$ participants reported death was more than $12.0 \%$ among infected individuals. When asked for death due to COVID-19 among different age groups, approximately three-fourth of the participants $(77.0 \%)$ reported that the individuals above 60 years of age were more prone to die due to COVID-19 infection. This was followed by $14.5 \%$ participants who reported that "both less than 10 years and more than 60 years" were prone to die. Very few $(0.6 \%)$ individuals reported that individuals below 18 years were prone to die. Only $1.0 \%$ stated that all groups were prone to die due to COVID-19.

\section{Groceries at home during the lockdown}

Almost one-third of the participants (36.3\%) had groceries for 8-15 days at home, followed by one-fourth participants (25.7\%) who had groceries for less than seven days. Around $15.0 \%$ participants had groceries for over 30 days at home, and the rest $22.5 \%$ had groceries for 16-30 days.

\section{Engagement in physical activities during the lockdown}

As depicted in Table 3, most participants were engaged in home-based exercise (39.0\%) and yoga-based activities $(28.0 \%)$. Cleaning, house chores and gardening were the house activities reported by $11.0 \%$ of the participants. Very few engaged in playing games $(4.0 \%)$ like badminton, cricket, football and indoor games. Few $(7.0 \%)$ relied on food-related practices like a healthy and balanced diet, diet control, vitamin and mineral supplements, drinking warm water, immunity improving diets for fitness. Nearly one-third $(32.0 \%)$ reported that they were not doing any physical activity during lockdown, while $64(4.0 \%)$ were engaged in healthcare and were still working.

\section{Entertainment during the lockdown}

Majority of the participants reported that they are using social media $(64.0 \%)$ and watching television $(63.0 \%)$ during the lockdown period as a means of entertainment. More than onethird participants $(37.0 \%$ ) informed that they were involved in indoor games like Ludo, board

\begin{tabular}{lr}
\hline List of activities & $n(\%)$ \\
\hline Home-based exercise (aerobics, app-based exercise, cycling, dance, indoor games, floor exercise, & $595(39.0 \%)$ \\
jogging, jumping, Pilates, running, skipping, walking and zumba) & $423(28.0 \%)$ \\
Yoga (yoga, meditation, pranayam/breathing exercise and suryanamaskar) & $170(11.0 \%)$ \\
House activities (cleaning, house decors and gardening) & $103(7.0 \%)$ \\
Diet based (healthy and balanced diet, diet control, vitamin and mineral supplements, warm & $57(4.0 \%)$ \\
water, immunity improving diets) & $64(4.0 \%)$ \\
Games (badminton, cricket, football and indoor games) & $489(32.0 \%)$ \\
Working in healthcare & Tactivities during \\
Nothing & lockdown \\
\hline
\end{tabular}


JHR

35,4

304

games, playing cards, chess and Xbox. Net-based streaming/videos/sites like Netflix, Amazon Prime videos, Hotstar, YouTube, TikTok, Voot, Zee5 were used by $28.0 \%$ of the participants during the lockdown. Spending time with family or talking on phone with family or friends was preferred by $22.0 \%$, while $19.0 \%$ engaged in cooking. The other activities included art-based activities $(9.0 \%)$ like art, dance, guitar, painting, singing, writing and reading $(5.0 \%)$. Only $2.0 \%$ participants were not doing anything for entertainment.

\section{Discussion}

The WHO declared COVID-19 as a pandemic on March 11, 2020, and there is scanty literature available of what the masses know this outbreak. This outbreak led to a feeling of uncertainty about what the future holds in terms of physical, economic, social and mental well-being. The present study aimed toward determining how well people in India know about COVID-19 outbreak and actions toward coping with the lockdown.

The demographic characteristics of the participants revealed an almost balanced distribution of both the genders. The mean age of the participants was 28.70 years, ranging from 18-84 years. Almost $73.0 \%$ of the participants were below 31 years, which was expected, given the online nature of this survey. The educational profile depicts that maximum participants held a bachelor's degree followed by a postgraduate degree. The maximum participants were healthcare workers, followed by students. Although there were 507 healthcare workers among the respondents, 64 were working at the time of this study. The lesser number of healthcare workers working at the time of this study could be due to the reason of closure of clinical services at outpatient departments, private clinics and dental clinics. When this study was conducted, only essential medical services and emergency medical services were functioning.

It was encouraging to note that in the present study, the participants were attentive and aware about the primary and secondary symptoms of COVID-19 and its spread through droplets from the nose or mouth. This is in line with the symptoms as documented by the WHO [7]. It is also evident that the participants are aware that the using multiple masks, smoking habits and consumption of alcohol are not effective against COVID-19. These points have been mentioned in the frequently asked questions (FAQs) of the WHO and MoHFW websites. The response to this question makes it evident that people are reading these awareness materials available.

Globally, the WHO and in India the MoHFW have emphasized on the importance of hand washing through several health-promotion campaigns and pamphlets. Several such awareness pamphlets, myth busters and FAQs are available on the WHO website [7]. These are also available in the national language Hindi to reach maximum audience [8]. These hand hygiene practices seem to have made people aware about its importance, as more than 95.0\% participants were aware. In addition, $80.0 \%$ participants felt that the steps taken by the government are effective, while $93.0 \%$ opined that lockdown would be effective. However, the participants were apprehensive about the infection and unsure of whether summer or high temperature to would contain the spread of infection.

Maximum individuals stated a credible medical source of information "WHO" as their primary source of information for COVID-19. Most of the participants referred to more than one resource for information. The questions related to fatality due to COVID-19 and age group most affected yielded variable responses. These findings are suggestive that there is a need to prioritize the information being given out in awareness campaigns to include information on mortality rate, recommended hand hygiene practices and reduced risk based on age.

The WHO has provided several recommendations for psychosocial and mental health during the COVID-19 outbreak. Some of these include avoiding reading/watching/listening to 
distressing news, amplifying positive and hopeful stories as well as staying in touch with family/friends through telephone/emails/social networks as well as encouraging healthy activities [11]. The MoHFW has also developed several videos on how to deal with health during the lockdown, which include tips for reducing and coping with anxiety and stress, taking care of children, as well as mental health [12].

During this period of lockdown, it is important to stay at home in a positive and cheerful manner, which is nevertheless a challenging task. A recent article on Harvard Health Blog has highlighted the importance of yoga, meditation and controlled breathing for coping with coronavirus anxiety [13]. In the present study, the participants engaged themselves in multiple form of either physical fitness activity like yoga, meditation, games, home-based exercises or entertaining themselves through social media, watching television, spending time with family and talking on phone. These activities were beneficial in coping with stress and anxiety. While $32.0 \%$ reported of not doing anything for physical fitness, only $2.0 \%$ reported not doing anything for keeping themselves entertained.

The present survey was conducted using a self-rated questionnaire using an online link; therefore, those who were interested might have only participated, which included a large number of healthcare workers and students. Thus, the generalizability of the findings is, therefore, limited. Limitations of an online survey such as lack of access to the internet were also the demerits in the present study. However, the survey had to be completed in a narrow timeline, given the fast-changing nature of such outbreaks. Also, the study was conducted in initial phase of COVID-19 outbreak in the Indian setting. This study might be useful in planning for development of an information, education and communication (IEC) strategy and materials to target the larger audience at the national and international level for behavior changes. The pandemic and the lockdown has led to long-term impact on all individuals; the findings of this study can help to better plan better physical and mental well-being. Future studies need to be aimed toward exploring the long-term effect of the lockdown and the pandemic.

\section{Conclusion}

The participants in the study seem to have good knowledge about COVID-19 infection, but certain beliefs need to be addressed. Correcting these beliefs should be targeted through information campaigns organized by government agencies, health professionals and media coverage. Mental health, as well as home-based physical activities, can also be focused through these health-promotion campaigns and information, education and counseling materials.

\section{References}

1. World Health Organization [WHO]. Statement on the second meeting of the International Health Regulations (2005) Emergency Committee regarding the outbreak of novel coronavirus (2019nCoV). Geneva: WHO; 2020.

2. World Health Organization [WHO]. WHO Director-General's opening remarks at the media briefing on COVID-19. 2020. [updated 2020 March 11; cited 2020 March 28]. Available from: https://www.who. $\mathrm{int} / \mathrm{dg} / \mathrm{speeches/detail/who-director-general-s-opening-remarks-at-the-media-briefing-on-covid-19-}$ 11-march-2020.

3. World Health Organization [WHO]. Coronavirus situation dashboard. 2020 [cited 2020 Apr 6]. Available from: https://who.sprinklr.com/.

4. World Health Organization [WHO]. Coronavirus disease (COVID-2019) India situation reports. 2020. [cited 2020 April 6]. Available from: https:/www.who.int/india/emergencies/india-situationreport.

5. United Nations. COVID-19: Lockdown across India, in line with WHO guidance. 2020. [updated 2020 March 24; cited 2020 April 6]. Available from: https://news.un.org/en/story/2020/03/1060132. 
JHR 35,4
6. Ministry of Health and Family Welfare Government of India. Detail question and answers on COVID-19 for public. 2020 [cited 2020 Apr 6]. Available from: https:/www.mohfw.gov.in/ index.html.

7. World Health Organization [WHO]. Coronavirus disease (COVID-19) advice for the public. 2020. [cited 2020 April 6]. Available from: https://www.who.int/emergencies/diseases/novel-coronavirus2019/advice-for-public.

8. Centers for Disease Control and Prevention [CDC]. Coronavirus (COVID-19): information for healthcare professionals. 2020. [cited 2020 April 6]. Available from: https://www.cdc.gov/ coronavirus/2019-nCoV/index.html.

9. Polit DF, Beck CT. The content validity Index: are you sure you know what's being Reported ? Critique and recommendations. Res Nurs Health. 2006; 29(5): 489-97. doi: 10.1002/nur.20147.

10. World Medical Association. World medical association declaration of Helsinki:ethical principles for medical research involving human subjects. JAMA. 2013; 310(20): 2191-94. doi: 10.1001/jama.2013.281053.

11. World Health Organization [WHO]. Mental health and psychosocial considerations during the COVID-19 outbreak. 2020 [cited 2020 Apr 6]. Available from: https://www.who.int/docs/defaultsource/coronaviruse/mental-health-considerations.pdf.

12. Ministry of Health and Family Welfare Government of India. Ministry of health and family Welfare. Government of India; 2020 COVID- 19 INDIA [cited 2020 Apr 6]. Available from: https:// www.mohfw.gov.in/.

13. Sharp J. Coping with coronavirus anxiety. Harvard Heal. Publ. 2020 [updated 2020 March 12; cited 2020 April 6]. Available from: https://www.health.harvard.edu/blog/coping-withcoronavirus-anxiety-2020031219183.

\section{Corresponding author}

Rohit Ravi can be contacted at: rohitravi94@gmail.com

For instructions on how to order reprints of this article, please visit our website: 